\title{
Numerical laminar forced convection modelling in a ceramic and concrete solar collector with non-circular duct
}

\author{
Tomasz Janusz, Teleszewski ${ }^{1{ }^{* *}}$ \\ ${ }^{1}$ Department of HVAC Engineering, Bialystok University of Technology, Bialystok 15-351, Poland
}

\begin{abstract}
The paper presents simulations of laminar forced convection in non-circular piping of flat solar collectors made of ceramics or concrete, which are characterized by a low thermal conduction coefficient. The cross-sections of piping in the shape of a regular polygonal, elliptic, superellipse (Lamé curve) and Cassini oval were used for the calculations. In order to perform the simulation, a simplified two-dimensional model of laminar forced convection in a straight axis duct was used and the issue of $\mathrm{H} 2$ (constant axial wall heat flux with uniform peripheral wall heat flux) for materials with low thermal conductivity was applied. The calculations were made using the boundary element method (BEM) in a calculation program written by Fortran by the author. In the work, the number of Poiseuille, Nusselt and dimensionless parameters for the evaluation of heat exchangers such as the area goodness factor and the volume goodness factor were determined in the function of characteristic geometrical parameters of the assumed cross-sectional shapes of the flat solar collector piping.
\end{abstract}

\section{Introduction}

Solar collectors are widely used in the production of domestic hot water, heating and supporting other heating devices such as heat pumps. The solar collector converts solar radiation into thermal energy and then transfers it to the heating system. In order to increase the thermal efficiency of the solar collector, it is possible to optimize the solar collector by modifying the elements of the solar collector, e.g. by application of an absorber with a high solar absorption coefficient [1], adding turbulators in the risers [2], changing the shape of the solar collector's cross-section [3], replacement of thermal insulation of the solar collector with thermal insulation with a lower thermal conductivity or increasing the thickness of the thermal insulation layer on the solar collector housing [4]. The shape of the cross-section of the piping plays an important role in the transfer of heat by convection forced from the walls of the risers to the working heating medium [3]. The most commonly used shape in solar collector risers is a circular pipe. Other shapes of ducts can also be found in the literature, e.g. a cross-section of rhombic [5], elliptical [6] or rectangle risers [7]. Another parameter affecting the heat transfer from the piping wall to the working

\footnotetext{
*Corresponding author: t.teleszewski@pb.edu.pl
} 
medium is the thermal conductivity of the material from which the riser tubes are made. Solar collectors are often used with a high thermal conductivity, i.e. piping is made of copper or aluminum. Currently, flat solar collectors have also appeared, whose piping materials are characterized by low thermal conductivity, i.e. ceramic and concrete flat solar collectors. Examples of constructions and studies on ceramic solar collectors can be found in the literature [8-11]. An exemplary cross-section by a prototype ceramic solar collector made at the Department of Heat Engineering, Heating and Ventilation of Białystok University of Technology is presented in Figure 1. Teleszewski [12] presents a comparison of non-circular solar collector pipes using the boundary element method. In paper [12], the problem designated as H1 was adopted, which is dedicated for risers with high thermal conductivity, where the boundary condition is a constant temperature at the perimeter of the duct cross-section and a constant axial heat flux.

In the case of ceramic and concrete flat plate collectors, where the thermal conductivity is small, according to the literature [13-15], the H2 problem should be adopted, in which the boundary condition is assumed as a constant axial wall heat flux density with uniform peripheral wall heat flux. The advantage of ceramic and concrete solar collectors is piping from ecological materials, that is concrete or ceramics.

The aim of this study is to compare the different cross-sectional shapes of the solar collector piping with a small thermal conductivity using classical dimensionless quantities such as the Poiseuille number, Nusselt number, area goodness factor and volume goodness factor. The parameters the area goodness factor and the volume goodness factor can be used to compare different heat exchangers [16-18]. The shapes of the cross-sections of the regular polygon, Cassini oval and superellipse (Lamé curve) were adopted for the calculations. The work also determined the practical formula of the Nusselt and Poiseuille numbers in function of geometric parameters of the presented shapes.

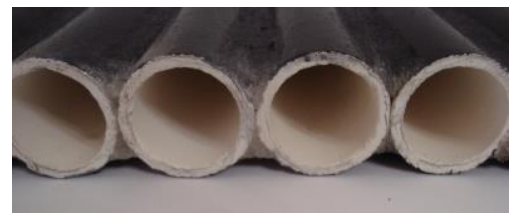

Fig. 1. Ceramic piping of the solar collector developed at the Bialystok University of Technology in the Department of HVAC Engineering.

\section{Problem description and mathematical formulation}

Figure 2 shows the calculation scheme and boundary conditions in the cross-section of the solar collector piping. In order to compare selected cross-sectional shapes of the solar collector piping with dimensionless quantities related to fluid flow and heat transfer, a simplified laminar forced convection model was adopted in straight ducts for a Newtonian fluid with a constant thermal conduction coefficient $k$ and constant dynamic viscosity $\mu$ for fully developed flow; in the body forces, viscous dissipation, and radiation heat transfer were neglected. For the above assumptions, forced convection for laminar flow in any cross-section of the straight duct is described by the continuity (1), momentum (2) and the energy equations (3):

$$
\frac{\partial u_{z}}{\partial z}=0
$$




$$
\begin{aligned}
\mu\left(\frac{\partial^{2} u_{z}}{\partial x^{2}}+\frac{\partial^{2} u_{z}}{\partial y^{2}}\right) & =\frac{\partial p}{\partial z}, \quad \frac{\partial p}{\partial x}=\frac{\partial p}{\partial y}=0 \\
k\left(\frac{\partial^{2} T}{\partial x^{2}}+\frac{\partial^{2} T}{\partial y^{2}}\right) & =\rho c_{p} \frac{\partial T}{\partial z} u_{z}
\end{aligned}
$$

where $u_{z}$ is the axial velocity, $T$ is temperature, $p$ is pressure, $c_{p}$ is specific heat capacity and $\rho$ is density.

In the case of condition $\mathrm{H} 2$ for walls constructed of materials with a low thermal conduction coefficient, the following Neumann boundary conditions are assumed:

$$
q_{w}=-k \frac{\partial T}{\partial n}, \text { at } L_{h}
$$

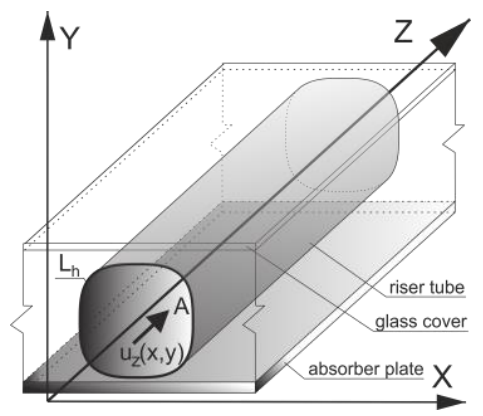

Fig. 2. Geometry and boundary conditions in laminar forced convection by the solar collector piping.

\section{Numerical results and discussion}

The equations of momentum (2) and energy (3) were determined by the boundary element method [19]. First, the velocity field from equation (2) was determined, which is a condition in the cross-section area $A$ for equation (3). A temperature field was determined from the energy equation (3). Details of the boundary element method algorithm for determining forced convection can be found in [19]. For the calculations, 4000 constant boundary elements on the $L_{h}$ boundary and 40602 triangular cells in the $A$ cross-section were used. The Poiseuille number is defined as a function of the Reynolds number and the coefficient of friction $f$ :

$$
P o=f \frac{\operatorname{Re}}{4}, \quad \operatorname{Re}=\frac{\rho u_{m} D_{h}}{\mu}, \quad D_{h}=\frac{4 A}{L_{h}}, \quad f=\frac{8 \tau_{w}}{\rho u_{m}^{2}}, \quad \mathrm{u}_{m}=\frac{1}{A} \iint_{A} u_{z} d A
$$

where $u_{m}$ is the average velocity, $D_{h}$ is the hydraulic diameter, $\boldsymbol{A}$ is the cross-sectional area of the duct, while $\tau_{w}$ is the peripherally averaged wall shear stress. The Nusselt number is described by the following relationship:

$$
\mathrm{Nu}=\frac{q_{w} D_{h}}{k\left(T_{w}-T_{b}\right)}, \quad T_{w}=\frac{1}{L_{h}} \int_{L_{h}} T_{w} d L_{h}, \quad T_{b}=\frac{1}{A u_{m}} \iint_{A} u_{z} T d A
$$


where $T_{w}$ is the average temperature at the boundary of $L_{h}, T_{b}$ is the bulk temperature and $q_{w}$ is the heat flux density.

In order to verify the BEM method, the results of numerical calculations with the analytical solution for the square duct $[13,14]$ were compared. The relative differences for the geometry adopted above did not exceed $0.001 \%$ for the Poiseuille number and $0.005 \%$ for the Nusselt number.

The following cross-section shapes were assumed for computer simulations, whose characteristic geometrical parameters $\sigma$ are described in Table 1: regular polygon, ellipse, superellipse with the assumption of equal minor to the major axis $(a / b=1)$, and Cassini oval.

Table 1. Description of the characteristic geometrical parameters of the assumed shapes of the cross-sections of the flat solar collector piping.

\begin{tabular}{|c|c|c|}
\hline $\begin{array}{c}\text { The shape of the cross section of the } \\
\text { solar collector piping }\end{array}$ & $\begin{array}{c}\text { Description of the geometric } \\
\text { factor } \boldsymbol{\sigma}\end{array}$ & Symbol of $\boldsymbol{\sigma}$ \\
\hline regular polygon & $\begin{array}{c}\text { number of sides of a regular } \\
\text { polygon }\end{array}$ & $n$ \\
\hline ellipse & $\begin{array}{c}\text { ratio of the minor to the major axis } \\
\text { for an ellipse }\end{array}$ & $A / B$ \\
\hline $\begin{array}{c}\text { superellipse with the assumption of equal } \\
\text { minor to the major axis }(a / b=1)\end{array}$ & exponent of the super ellipse & $e$ \\
\hline Cassini oval & ratio of the coefficients C and D & $C / D$ \\
\hline
\end{tabular}

The method of determining the coordinates of the Cassini oval is presented in [12], while the superellipse is described by the following formula:

$$
\left|\frac{x}{a}\right|^{e}+\left|\frac{y}{b}\right|^{e}=1
$$

The result of computer simulations is a formula showing the dependence of the Poiseuille number on the superellipse and the Nusselt number for the $\mathrm{H} 2$ problem for the shape of a regular polygon, elliptical, Cassini oval and superellipse in function of the characteristic geometric parameters $\sigma$.

$$
\begin{aligned}
& P o(\sigma), N u(\sigma)=\frac{a+b \sigma^{0.5 m}+c \sigma^{m}+d \sigma^{1.5 m}+e \sigma^{2 m}}{1+f \sigma^{0.5 m}+g \sigma^{m}+h \sigma^{1.5 m}+i \sigma^{2 m}}, \\
& \sigma=m \vee A / B \vee C / D \vee e, \quad 0.5 \leq e \leq 4, \quad A / B \geq 0.05
\end{aligned}
$$

where $a, b, c, d, e, f, g, h, i$, and $m$ are coefficients shown in Table 2. The correlation coefficient for formula $(8)$ is equal to $R^{2} \approx 1$. The dependences of the Poiseuille number as a function of geometric parameters for the regular polygon, ellipse and Cassini oval are presented in [12].

Figure 3a presents a comparison of the results of calculations of Nusselt numbers for the problem $\mathrm{H} 1$ [12] and $\mathrm{H} 2$ as a function of geometrical parameters for the cross-section shape of a regular polygon, elliptical shape, and Cassini oval. Figure 4 shows the Poiseuille and Nusselt numbers in function $e$ for two $\mathrm{H} 1$ and $\mathrm{H} 2$ problems for the shape of a superellipse, assuming that the minor and major axes are equal. In the case of a circular tube, the values of the Nusselt number for the problem $\mathrm{H} 1$ and $\mathrm{H} 2$ are equal to each other due to the lack of sharp edges in the circle [10]. In the case of cross-sectional non-circular shapes, the Nusselt trend for $\mathrm{H} 1$ and $\mathrm{H} 2$ can be divergent, e.g. for an elliptical cross-section the Nusselt number 
decreases with the increase of the geometric parameter $\sigma$ for the H1 case, while for the $\mathrm{H} 2$ case the Nusselt number increases with the increase of $\sigma$. The maximum value of the Nusselt number $(\mathrm{Nu}=5.11)$ for selected cross-sections of risers for the $\mathrm{H} 2$ problem has a Cassini oval cross-section for $\sigma=0.975$.

Table 2. Equation coefficients (8) for determining the Poiseuille and Nusselt numbers for selected shapes of the flat solar collector.

\begin{tabular}{|c|c|c|c|c|c|}
\hline \multicolumn{6}{|c|}{ Coefficients of equation (8) to determine the Poiseuille number } \\
\hline shape & $\mathrm{a}$ & $\mathrm{b}$ & $\mathrm{c}$ & $\mathrm{d}$ & $\mathrm{e}$ \\
\hline superellipse & -2.9446 & 15.449 & -13.308 & 17.121 & -0.902 \\
\hline & $\mathrm{f}$ & $\mathrm{g}$ & $\mathrm{h}$ & $\mathrm{i}$ & $\mathrm{m}$ \\
\hline superellipse & -1.089 & 0.4884 & 0.6941 & -0.0158 & 2 \\
\hline Coefficients of equation (8) to determine the Nusselt number (H2 case) \\
\hline & $\mathrm{a}$ & $\mathrm{b}$ & $\mathrm{c}$ & $\mathrm{d}$ & $\mathrm{e}$ \\
\hline polygonal & 112.85 & -13.32 & -46.019 & 13.572 & 0 \\
\hline ellipse & 0.0768 & -2.376 & 108.44 & 14.12 & 0 \\
\hline Cas. oval & 4.3636 & -7.1784 & -1.709 & 8.148 & -3.6049 \\
\hline superellipse & -34.482 & 197.466 & -393.07 & 258.1875 & 46.06 \\
\hline & $\mathrm{f}$ & $\mathrm{g}$ & $\mathrm{h}$ & $\mathrm{i}$ & $\mathrm{m}$ \\
\hline polygonal & 7.917 & -10.6029 & 3.1102 & 0 & 2 \\
\hline ellipse & 2.609 & 16.034 & 7.918 & 0 & 2 \\
\hline Cas. oval & -1.6514 & -0.0563 & 1.14904 & -0.4364 & 4 \\
\hline superellipse & 32.3 & -60.297 & 34.66 & 16.423 & 2 \\
\hline
\end{tabular}

a)

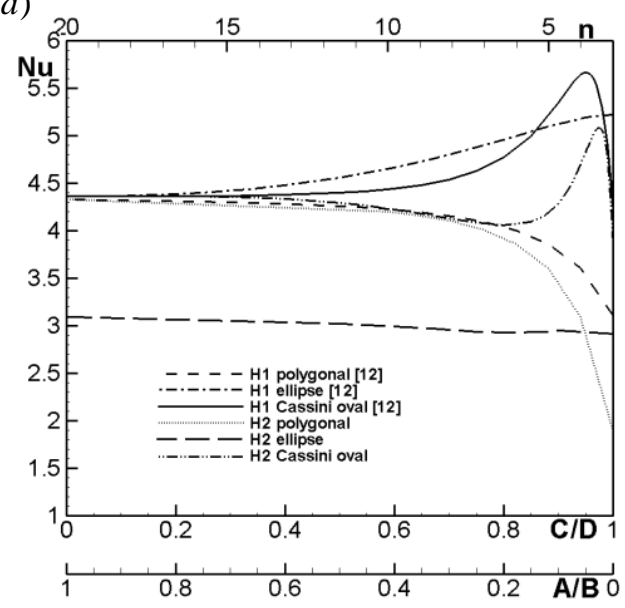

b)

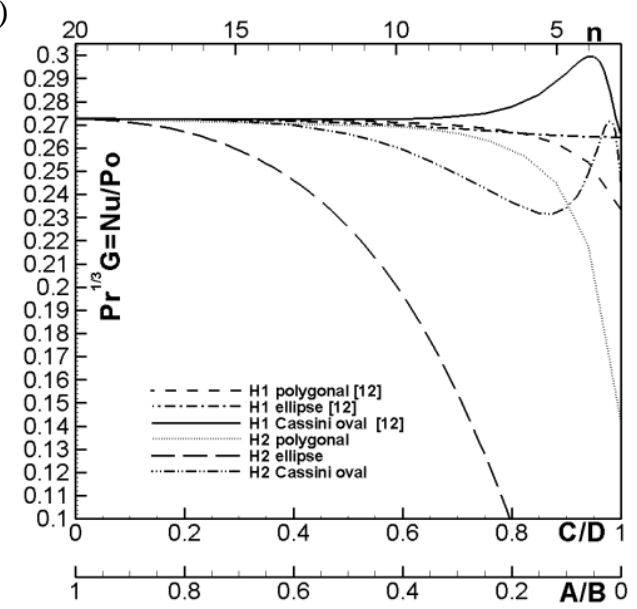

Fig. 3. Comparison of Nusselt numbers (a) and the area goodness factor (b) for the $\mathrm{H} 1$ and $\mathrm{H} 2$ cases for the cross-sections with the shape of a regular polygon, elliptic and Cassini oval. 
In order to compare the selected duct shapes, the area goodness factor $G$ and the volume goodness factor VGF were used. In the classical definition, the area goodness factor is a function of the Colburn factor $j$ and the Fanning friction factor $f$, but in the case of fully developed forced convection through a straight duct the Poiseuille and Nusselt numbers are independent of the Prandtl number, so it is possible to use a simplified form as a ratio of the Nusselt and Poiseuille numbers [2]:

$$
\mathrm{G}=\frac{j}{f}=\frac{\mathrm{Nu}}{\operatorname{Pr}^{1 / 3} \mathrm{Po}} \Rightarrow \operatorname{Pr}^{1 / 3} \mathrm{G}=\frac{\operatorname{Pr}^{1 / 3} j}{f}=\frac{\mathrm{Nu}}{\mathrm{Po}}, \quad \operatorname{Pr}=\frac{c_{p} \mu}{k}
$$

where St is the Stanton number. In the assessment of heat exchangers, a higher area goodness factor means a lower flow area, therefore a higher value of area goodness factor is desired [13]. Figure $3 \mathrm{~b}$ shows the comparison of the area goodness factor as a function of the geometric coefficient $\sigma$ for the shapes of a regular polygon, elliptic and Cassini oval, while Figure 4 shows the area goodness factor for a superellipse. In the case of cross-sections of non-circular shapes, the trends of area goodness factors for cases $\mathrm{H} 1$ and $\mathrm{H} 2$ are similar, while the numerical values of area goodness factors for the $\mathrm{H} 2$ issue are much lower than for the case of H1. The largest value of area goodness factor $(\mathrm{Nu} / \mathrm{Po}=0.27)$ for the $\mathrm{H} 2$ issue is equal to the shape of the Cassini oval with the geometric parameter $\sigma=0.975$.

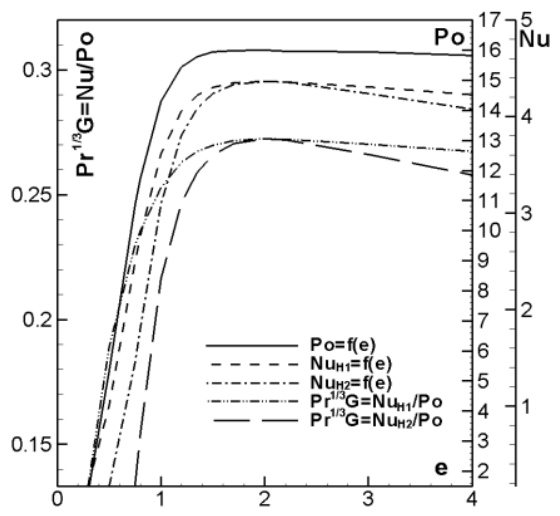

Fig. 4. The Poiseuille number and the comparison of Nusselt numbers and the volume goodness factor of a superellipse for $\mathrm{H} 1$ and $\mathrm{H} 2$.

The second method of comparing selected shapes is to use the volume goodness factor VGF, which is defined by the following relationship:

$$
V G F=\frac{\mathrm{St}}{f^{1 / 3}}, \quad \mathrm{St}=\frac{\mathrm{Nu}}{\mathrm{RePr}}
$$

A high volume goodness factor requires a small volume to demonstrate the same performance in terms of heat transfer rate [13, 18]. To determine the volume goodness factor as a function of geometric factors, $50 \%$ propylene glycol mixtures were assumed, the properties of which are presented in Table 3 [20]. Figure 5 shows the comparison of the volume goodness factor for the $\mathrm{H} 1$ and $\mathrm{H} 2$ cases for the following solar collector pipes: square $(\sigma=4)$, circle $(\sigma=1000)$, Cassini oval $(\sigma=0.945)$ and superellipse $(\sigma=4)$. Analyzing the obtained VGF results, it can be seen that the volume goodness factor values, 
as in the case of the area goodness factor for the $\mathrm{H} 2$ issue, are lower than the VGF value for the $\mathrm{H} 1$ issue. For example, in the case of a superellipse cross-section with a geometric coefficient of $\sigma=4$, VGF for the H2 problem is $3.5 \%$ smaller than the VGF for the $\mathrm{H} 1$ issue, while for a square shape $(\sigma=4) \mathrm{VGF}$ for issue H2, it is $14.3 \%$ smaller than VGF for H1. Differences between VGF values for H2 and H1 are greater for cross-section shapes that are characterized by a greater number of sharp edges or corners. In the case of a circular cross-section, VGF is equal for both $\mathrm{H} 2$ and $\mathrm{H} 1$. For selected cross-sectional shapes for the H2 issue, the highest VGF value is equal to a Cassini oval shape with a geometric coefficient equal to $\sigma=0.975$ and it is smaller by $9.9 \%$ than the maximum VGF value for the Cassini oval cross-sectional shape $(\sigma=0.945)$ [12] for the H1 issue.

Table 3. Physical quantities for $50 \%$ propylene glycol mixtures [20].

\begin{tabular}{|c|c|c|c|}
\hline Description & Symbol & Value & Unit \\
\hline $\begin{array}{c}\text { Dynamic viscosity of the working } \\
\text { medium in the solar collector }\end{array}$ & $\mu$ & 0.002 & $\mathrm{~kg} / \mathrm{m} / \mathrm{s}$ \\
\hline $\begin{array}{c}\text { Thermal conductivity of the working } \\
\text { medium in the solar collector }\end{array}$ & $k$ & 0.4 & $\mathrm{~W} / \mathrm{m} / \mathrm{K}$ \\
\hline $\begin{array}{c}\text { Density of the working medium in the } \\
\text { solar collector }\end{array}$ & $\rho$ & 1025 & $\mathrm{~kg} / \mathrm{m}^{3}$ \\
\hline $\begin{array}{c}\text { Specific heat capacity of the working } \\
\text { medium in the solar collector }\end{array}$ & $c_{p}$ & 3480 & $\mathrm{~J} / \mathrm{kg} / \mathrm{K}$ \\
\hline
\end{tabular}

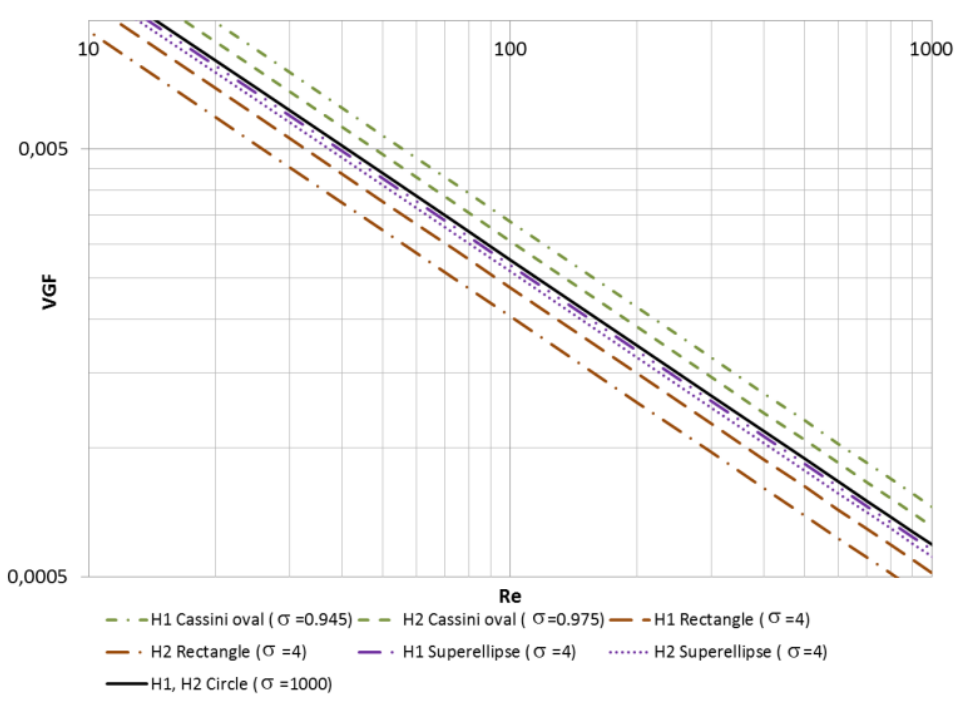

Fig. 5. Comparison of the volume goodness factor for the $\mathrm{H} 1$ and $\mathrm{H} 2$ cases for selected solar collector risers.

\section{Conclusions}

This paper presents a comparison in the aspect of forced convection of selected cross-sections of risers of a flat solar collector made of ceramic or concrete, which is characterized by a low thermal conduction coefficient. It should be noted here that the construction of solar collectors with ceramic or concrete piping is used rarely compared to solar collectors in which the piping is made of materials with a high thermal conductivity coefficient, such as copper or aluminum. A simplified model of laminar forced convection 
was adopted for the fully developed flow in a straight axis duct for the H2 case, which is dedicated for materials with low thermal conductivity. The selected cross-sectional shapes were compared using the classic methods of area goodness factor and volume goodness factor. Based on the results of simulations made with the boundary elements method for selected cross-section shapes, the optimal solution is Cassini's oval for the aspect ratio $\sigma=0.975$. It should be noted here that the methods area goodness factor and volume goodness factor are used for initial evaluation of heat exchangers, while a full assessment of thermal efficiency of heat exchangers should be done by experiment.

The study has been implemented from the resources financed by the Ministry of Science and Higher Education in Poland (WZ/WBiIS/9/2019).

\section{References}

1. D. Mercs, A. Didelot, F. Capon, J.-F. Pierson, B. Hafner, A. Pazidis, S. Föste, R. Reineke-Koch, Energy Procedia 91, 84 (2016)

2. S. Bhattacharyya, H. Chattopadhyay, A. Haldar, Beni-Seuf Univ. J. Appl. Sci. 7, 118 (2018)

3. E. Ekramian, S. G. Etemad, M. Haghshenasfard, J. of Fluid Flow, Heat and Mass Transfer 1, 1 (2014)

4. K. Pandecha, A. Pongtornkulpanich, S. Sukchai, T. Suriwong, Journal of Renewable Energy and Smart Grid Technology 10, 27 (2015)

5. H. Taherian, E. Yazdanshenas, In: Proceedings of the Second International Green Energy Conference, IGEC2-126 (2006)

6. K. Sivakumar, N. Krishna Mohan, B. Sivaraman, Indian J. Sci. Technol. 4, 4 (2011)

7. A. Kumar, Energy Procedia 57, 2102 (2014)

8. Y. Yang, S. Cao, J. Xua, B. Cai, Ceram. Int. 39, 6009 (2013)

9. Y. Yang, Q. Wang, D. Xiu, Z. Zhao, Q. Sun, Energy Build. 62, 15 (2013)

10. X.-Y. Sun, X.-D. Sun, X.-G. Li, Z.-Q. Wang, J. He, B.-S. Wang, Energy Build. 75, 176 (2014)

11. M. Zukowski, G. Woroniak, Solar Energy 146, 532 (2017)

12. T. J. Teleszewski, E3S Web Conf. 22, 00177 (2017)

13. R. K. Shah, A. L. London, Laminar Flow Forced Convection in Ducts, Advanced in Heat Transfer (Academic Press, London, UK, 1978)

14. G. L. Morini, Int. J. Heat Mass Transf. 43, 741 (200)

15. S. Kakac, Y. Yener, A. Pramuanjaroenkij, Convective Heat Transfer ( $^{\text {rd }}$ ed., CRC Press, NY, 2013)

16. J.-H. Doo, H.S. Yoon, M. Y. Ha, Transactions of the Korean Society of Mechanical Engineers B 33, 6 (2009)

17. S. Ray, D. Misra, Int. J. Therm. Sciences, 49 (2010)

18. S. W. Hwang, D. H. Kim, J. K. Min, J. H. Jeong, J. Mech. Sci. Technol. 26, 2949 (2012)

19. T. J. Teleszewski, S. A. Sorko, International Journal of Numerical Methods for Heat \& Fluid Flow 28, 1, 220 (2018)

20. M. J. Assael, E. Charitidou, S. Avgoustiniatos, W. A. Wakeham, Int. J. Thermophys. 10, 6, 1127 (1989) 